\title{
An evaluation of the Monarch chemistry analyser
}

\author{
D. J. Berry and C. P. Price \\ Department of Clinical Biochemistry, Addenbrooke's Hospital, Hills Road, \\ Cambridge CB2 2QR, UK
}

The Monarch Chemistry system, a centrifugal analyser incorporating sophisticated robotics for analytical rotor transfer and flexible software for workload scheduling, has been evaluated. The optical system is capable of monitoring absorbance, fluorescence and light scattering reactions. In addition, an ion selective electrode unit may be incorporated for the measurement of sodium, potassium and chloride.

The precision, accuracy, linearity, calibration stability and carry-over were investigated for 19 routine chemistries. The within-batch and between-day precision data were good in the majority of cases; some chemistries demonstrated poor performance at low analyte concentrations. Method comparison studies showed good agreement, with small discrepancies being due to different calibration material and methodological differences. Major discrepancies were found with $C K$ and $L D$; linearity studies were good in all cases, except calcium. No significant sample or reagent carry-over was found.

Assessment of throughput for a variety of test profiles varied between 300 and 605 tests per hour.

The instrument was easy to operate, very flexible and capable of handling a large and varied workload.

\section{Introduction}

The centrifugal analyser was first described by Dr Norman Anderson in 1969 [1]. The first commercial instrument was released in 1978 and since then several instruments have been developed based on this technology [2]. The recent development of the Monarch chemistry analyser features a number of new innovations in line with predictions made by Tiffany [3]. To virtually eliminate operator intervention, the analyser incorporates a transport arm to transfer analytical rotors from the feed-stack through loading, analysis and finally to the discard stack. The optical system is capable of changing optical filters in less than $5 \mathrm{~s}$, enabling more than one chemistry to be run within the same rotor. In addition, the system provides a sophisticated software package which includes an intelligent work scheduling system.

The analytical performance of the Monarch was assessed for 19 different analytes. The aspects evaluated included within-day and between-day precision at three different analyte concentrations; calibration stability; method linearity; relative accuracy and system carry-over [4].

\section{Materials and methods}

The instrument

The Monarch is a single free-standing unit requiring a 13 amp power supply; diluent and waste bottles are selfcontained within the system. The instrument is capable of performing up to a maximum of 24 tests per sample. An optional ISE unit allows the measurement of sodium, potassium and chloride, which are always made together. The sample throughput for a variety of chemistry profiles varies between 300 and 605 tests per hour. Stat requests can be processed at any time during a routine run; when completed the instrument returns to the original request. The optical system allows the measurement by absorbance, fluorescence and light-scattering techniques. Either rate or end-point assays, with up to four reagent additions, can be monitored.

Samples and reagents are housed in a compartment maintained at a temperature of $15^{\circ} \mathrm{C}$ for greater reagent stability. Reagent boats are identified by a bar-coded label which is read by an optical bar-code sensor.

Sample and reagent are loaded into a disposable UVT rotor via two stainless-steel pipette tips attached to the pipette arm. Each rotor contains 39 cuvettes into which sample (89 $\mu \mathrm{l}$ maximum) and reagent $(100-236 \mu \mathrm{l})$ are dispensed. The pipette arm is located in a thermal box, allowing movement between the reagent compartment and analysis compartment. The instrument uses two diluent-filled syringes to load the rotor. During loading the syringes are drawn down, resulting in sample and reagent being taken up into the tubing connected to the pipette tips. After sampling, the pipette arm moves back to the home position and sample and reagent are dispensed into the rotor. The pipette arm is heated to the same temperature as the analysis compartment (25, 30 or $\left.37^{\circ} \mathrm{C}\right)$.

The analysis compartment contains a robotic transport arm surrounded by a feed-stack, a loading table, analysis table and discard/park table. The feed-stack contains a supply of clean rotors. The optics module contains the tungsten and xenon lamps, a scanning monochromator and sets of mirrors, lenses and optical windows. Wavelengths between 340 and $690 \mathrm{~nm}$ can be selected.

The transport arm transfers the loaded rotor to the analysis table, where reagents and sample are mixed and data acquisition takes place. When the analysis is complete a full rotor is discarded and a partially used rotor is held on the park station, if possible, for re-use. 
The ISE module is housed independently within the system. Ion-selective electrodes determine the concentration of sodium, potassium and chloride in plasma, serum, urine or sweat. The sample is diluted, mixed and then drawn into the electrode module, which contains the sodium, potassium, chloride and reference assemblies.

The analyser is controlled by a computer with which the operator communicates via a keyboard and VDU. Two disks store test parameters, response data, results, user file data, utilities and diagnostic information.

The software contains a number of features which allow the user to review, edit and print information. Each test has a corresponding parameter table which may be readily accessed by the user. New tests may also be created in this way.

The workload may be scheduled in one of two ways either time-optimized or patient priority. In time-optimized mode the instrument schedules the request for optimum throughput by analysing batches of chemistries together. This is the most time-efficient and economical mode of operation. In patient priority mode, the system will schedule to analyse the tests sample by sample.

To increase the efficiency of the instrument and allow more than one chemistry to be run on one rotor, tests are assigned to a compatibility class. For tests to be in the same compatibility class the main characteristics of the class must be the same.

\section{Analytical methods and reagents}

Details of the methods employed in the evaluation are shown in table 1. All reagents were prepared and used according to the manufacturers' recommendations. The calibrators used were RefIL A, B, C (Instrumentation Laboratory) and for the calibration of BCP albumin, Nycomed Reference Material. A range of quality-control sera were used: Serachem Level I and II (Fisher Diagnostics), Technicon Reference (Technicon Instruments), Autonorm Low and High (Nycomed) and Precinorm E and Precipath E (Boehringer Mannheim)

\section{Precision}

The within-batch precision was assessed by analysing control sera at three different analyte concentrations. Eighteen samples of each control sera were assayed, thus ensuring that all samples would be analysed within one rotor. This was carried out using the instrument in both 'time-optimized' and 'patient-priority' mode.

The between-batch precision was assessed over a period of 20 working days. Quality-control material was reconstituted at the beginning of each day.

\section{Calibration stability}

The instrument was calibrated at the start of each day, according to the manufacturers' recommendations. Calibrators and control sera were then assayed immediately following calibration and at the end of each day.

\section{Linearity}

Samples known to contain high levels of analyte were diluted in varying proportions with $60 \mathrm{~g} / \mathrm{l} \mathrm{BSA}$. Where this was not possible a commercial lyophilized quality control material was reconstituted in a smaller volume than recommended to give elevated analyte concentrations. For the electrolytes, a stock solution of sodium

Table 1. Details of methods employed on Monarch and comparison system.

\begin{tabular}{|c|c|c|c|}
\hline Analyte & $\begin{array}{l}\text { Sample volume } \\
(\mu l)\end{array}$ & Principle of Monarch method & Comparison method \\
\hline \multirow{4}{*}{$\begin{array}{l}\text { Sodium } \\
\text { Potassium } \\
\text { Chloride } \\
\mathrm{TCO}_{2}\end{array}$} & & \multirow[t]{3}{*}{ ISE } & \multirow{3}{*}{$\begin{array}{l}\text { Flame photometry } \\
\qquad(\mathrm{Na}, \mathrm{K} \text { only) SMAII }\end{array}$} \\
\hline & 30 & & \\
\hline & & & \\
\hline & 3 & $\begin{array}{l}\text { Enzymatic, phosphoenolpyruvate } \\
\text { carboxylase }\end{array}$ & Indicator dye, SMAII \\
\hline Glucose & 3 & $\begin{array}{l}\text { Hexokinase, glucose-6-phosphate } \\
\text { dehydrogenase }\end{array}$ & Glucose oxidase SMAII \\
\hline Urea & 3 & Urease/GLDH & Diacetyl monoxime SMAII \\
\hline Greatinine & 9 & Picric acid & Jaffe SMAII \\
\hline Total protein & 5 & Biuret & Biuret SMAII \\
\hline Albumin & 3 & Bromocresol purple & Bromocresol purple SMAII \\
\hline Calcium & 5 & Cresolphthalein complexone & Cresolphthalein complexone SMAII \\
\hline Phosphate & 4 & Ammonium molybdate & Phosphomolybdate, SMAII \\
\hline Bilirubin & 8 & Sulphanilic acid & Jendrassik and Grof, SMAII \\
\hline ALP & 10 & $\begin{array}{l}\text { p-nitrophenyl phosphate with DEA } \\
\text { buffer }\end{array}$ & $\begin{array}{l}\text { p-nitrophenyl phosphate with AMP } \\
\text { buffer, SMAII }\end{array}$ \\
\hline ALT & 20 & L-alanine, $\alpha$-ketoglutarate & L-alanine optimized SMAII \\
\hline GK & 10 & Creatine phosphate & $\begin{array}{l}\text { Creatine phosphate, } \\
\text { Multistat III }\end{array}$ \\
\hline LD & 5 & Pyruvate $\rightarrow$ lactate & Pyruvate $\rightarrow$ lactate, Multistat III \\
\hline Urate & 20 & Uricase, $340 \mathrm{~nm}$ & Uricase $340 \mathrm{~nm}, \mathrm{RA} 1000$ \\
\hline Cholesterol & 3 & Cholesterol oxidase & Cholesterol oxidase, RA1000 \\
\hline Triglyceride & 3 & Lipase, glycerophosphate oxidase & Lipase, glycerophosphate oxidase \\
\hline
\end{tabular}


chloride was used for sodium and chloride and a solution of potassium chloride for potassium. Further dilutions of the stock were made in deionized water.

\section{Accuracy}

At least 100 patient samples were analysed on the Monarch and the results compared to those obtained in the routine laboratory. Details of comparison methods are given in table 1 .

\section{Carry-over}

The design of the instrument required investigation of both 'sample to sample' carry-over and 'reagent to sample or reagent' carry-over.

\section{Sample to sample carry-over}

Three sequences of a high pool followed by a low pool were assayed for each analyte. The mean carry-over was calculated for each sequence using the formula:

$$
\frac{L_{1}-L_{3}}{H_{3}-L_{3}} \times 100(\%)
$$

\section{Reagent to sample or reagent carry-over}

A mid-level human serum pool was assayed, in triplicate, such that, ultimately each chemistry had been preceded and followed by the other. The coefficient of variation for the analyte being investigated was then calculated.

\section{Results and discussion}

\section{Precision}

Results for the within-batch and between-day precision are shown in tables 2 and 3 respectively.

The results for the within-batch precision shows good performance in the majority of cases. The disappointing precision obtained at low levels of urea, ALT and triglyceride may be due to the low absorbance changes for these assays. Generally, the precision obtained whilst scheduling the instrument in 'patient-priority' mode was inferior to that found in time-optimized mode. This may be due to each test having its own blank in patientpriority mode, as opposed to one blank for a batch of tests in time-optimized mode.

The day-to-day precision was found to be acceptable, with the exception of creatinine, calcium, CK and triglyceride, which were disappointing. The performance of the $\mathrm{TCO}_{2}$ method was disappointing throughout the concentration range.

There was a definite improvement in the precision obtained for $\mathrm{CK}$ and $\mathrm{LD}$ when using alternative qualitycontrol material.

The performance of the ISE unit was very good.

\section{Calibration stability}

Assaying quality control material at the beginning and end of each day showed deterioration in the performance of some analytes. In these cases more frequent calibration may be required.
Table 2. Within-batch precision at three analyte concentrations. Results for time-optimized and patient-prioritized mode (in parentheses).

\begin{tabular}{|c|c|c|c|c|}
\hline Analyte & Mean & $S D$ & $C V(\%)$ & $C V(\%)$ \\
\hline \multirow[t]{3}{*}{ Sodium (mmol/l) } & $123 \cdot 6$ & $0 \cdot 88$ & 0.72 & $(0 \cdot 34)$ \\
\hline & $139 \cdot 1$ & $0 \cdot 66$ & $0 \cdot 48$ & $(0 \cdot 49)$ \\
\hline & $147 \cdot 9$ & 0.59 & $0 \cdot 40$ & $(0 \cdot 37)$ \\
\hline \multirow[t]{3}{*}{ Potassium (mmol/l) } & 1.90 & $0 \cdot 00$ & $0 \cdot 00$ & - \\
\hline & $4 \cdot 30$ & $0 \cdot 00$ & 0.00 & $(1 \cdot 18)$ \\
\hline & $6 \cdot 53$ & 0.05 & 0.69 & $(0 \cdot 76)$ \\
\hline \multirow[t]{3}{*}{ Chloride (mmol/l) } & $91 \cdot 0$ & $0 \cdot 56$ & $0 \cdot 62$ & $(0 \cdot 29)$ \\
\hline & $98 \cdot 7$ & $0 \cdot 38$ & $0 \cdot 38$ & $(0 \cdot 46)$ \\
\hline & $116 \cdot 5$ & $0 \cdot 36$ & $0 \cdot 31$ & $(0 \cdot 37)$ \\
\hline \multirow[t]{3}{*}{$\mathrm{TCO}_{2}(\mathrm{mmol} / \mathrm{l})$} & $15 \cdot 8$ & $0 \cdot 39$ & $2 \cdot 47$ & $(3 \cdot 72)$ \\
\hline & $25 \cdot 4$ & $0 \cdot 39$ & 1.55 & $(4 \cdot 71)$ \\
\hline & $27 \cdot 2$ & $0 \cdot 67$ & $2 \cdot 47$ & $(4 \cdot 57)$ \\
\hline \multirow[t]{3}{*}{ Glucose $(\mathrm{mmol} / \mathrm{l})$} & $4 \cdot 09$ & $0 \cdot 04$ & $1 \cdot 07$ & $(1.96)$ \\
\hline & $12 \cdot 30$ & $0 \cdot 14$ & $1 \cdot 12$ & $(2 \cdot 17)$ \\
\hline & $19 \cdot 79$ & $0 \cdot 20$ & $1 \cdot 01$ & $(1 \cdot 86)$ \\
\hline \multirow[t]{3}{*}{ Urea $(\mathrm{mmol} / \mathrm{l})$} & $5 \cdot 52$ & $0 \cdot 20$ & 3.55 & $(3.94)$ \\
\hline & $18 \cdot 22$ & $0 \cdot 26$ & $1 \cdot 42$ & $(2 \cdot 79)$ \\
\hline & $19 \cdot 11$ & $0 \cdot 35$ & $1 \cdot 19$ & $(3 \cdot 68)$ \\
\hline \multirow[t]{3}{*}{ Greatinine $(\mu \mathrm{mol} / \mathrm{l})$} & $101 \cdot 9$ & $2 \cdot 06$ & $2 \cdot 02$ & $(3 \cdot 01)$ \\
\hline & $452 \cdot 6$ & $4 \cdot 10$ & $0 \cdot 91$ & $(2 \cdot 21)$ \\
\hline & $713 \cdot 8$ & $5 \cdot 25$ & $0 \cdot 74$ & $(2 \cdot 40)$ \\
\hline \multirow[t]{2}{*}{ Total protein $(\mathrm{g} / \mathrm{l})$} & $40 \cdot 9$ & $0 \cdot 48$ & $1 \cdot 17$ & $(2 \cdot 58)$ \\
\hline & $67 \cdot 6$ & $0 \cdot 78$ & $1 \cdot 15$ & $(2 \cdot 05)$ \\
\hline \multirow[t]{3}{*}{$\operatorname{Albumin}(\mathrm{g} / \mathrm{l})$} & $24 \cdot 3$ & $0 \cdot 34$ & $1 \cdot 42$ & - \\
\hline & $38 \cdot 0$ & $0 \cdot 23$ & $0 \cdot 60$ & $(1 \cdot 68)$ \\
\hline & $49 \cdot 2$ & $0 \cdot 25$ & 0.51 & $(1 \cdot 29)$ \\
\hline \multirow[t]{3}{*}{ Calcium (mmol/l) } & 1.02 & $0 \cdot 02$ & $1 \cdot 63$ & - \\
\hline & $2 \cdot 42$ & $0 \cdot 03$ & $1 \cdot 63$ & - \\
\hline & $3 \cdot 19$ & $0 \cdot 02$ & $0 \cdot 66$ & $(1 \cdot 38)$ \\
\hline \multirow[t]{3}{*}{ Phosphate $(\mathrm{mmol} / \mathrm{l})$} & $1 \cdot 17$ & 0.02 & $1 \cdot 36$ & $(3 \cdot 16)$ \\
\hline & $1 \cdot 67$ & $0 \cdot 02$ & $1 \cdot 04$ & $(2 \cdot 49)$ \\
\hline & $2 \cdot 84$ & 0.02 & $0 \cdot 70$ & $(2 \cdot 48)$ \\
\hline \multirow[t]{3}{*}{ T Bilirubin $(\mu \mathrm{mol} / \mathrm{l})$} & $11 \cdot 9$ & $0 \cdot 24$ & $2 \cdot 01$ & $(2 \cdot 23)$ \\
\hline & $64 \cdot 9$ & $2 \cdot 61$ & $4 \cdot 03$ & $(3 \cdot 20)$ \\
\hline & $147 \cdot 7$ & $0 \cdot 61$ & $0 \cdot 41$ & $(1 \cdot 38)$ \\
\hline \multirow[t]{3}{*}{$\operatorname{ALP}($ I.U./l) } & $74 \cdot 3$ & $0 \cdot 98$ & $1 \cdot 32$ & $(3 \cdot 49)$ \\
\hline & $170 \cdot 8$ & $1 \cdot 39$ & $0 \cdot 81$ & $(2 \cdot 11)$ \\
\hline & $644 \cdot 7$ & $6 \cdot 00$ & 0.93 & $(1 \cdot 66)$ \\
\hline \multirow[t]{3}{*}{ ALT (I.U./l) } & $17 \cdot 7$ & 0.72 & $4 \cdot 06$ & $(6 \cdot 12)$ \\
\hline & $74 \cdot 7$ & $0 \cdot 81$ & 1.09 & $(1 \cdot 67)$ \\
\hline & $189 \cdot 0$ & $1 \cdot 10$ & 0.58 & $(1 \cdot 86)$ \\
\hline \multirow[t]{3}{*}{ GK (I.U./l) } & $121 \cdot 2$ & $1 \cdot 59$ & $1 \cdot 31$ & $(1 \cdot 88)$ \\
\hline & $245 \cdot 6$ & $3 \cdot 08$ & $1 \cdot 25$ & - \\
\hline & $411 \cdot 4$ & $6 \cdot 56$ & $1 \cdot 59$ & $(7 \cdot 88)$ \\
\hline \multirow[t]{3}{*}{ LD (I.U./l) } & $228 \cdot 9$ & $4 \cdot 85$ & $2 \cdot 12$ & $(3.49)$ \\
\hline & $370 \cdot 4$ & $5 \cdot 76$ & 1.55 & - \\
\hline & $622 \cdot 4$ & $5 \cdot 54$ & $0 \cdot 89$ & $(1 \cdot 76)$ \\
\hline \multirow[t]{3}{*}{ Urate $(\mu \mathrm{mol} / \mathrm{l})$} & $200 \cdot 8$ & $2 \cdot 16$ & $1 \cdot 07$ & $(1 \cdot 76)$ \\
\hline & $327 \cdot 0$ & $5 \cdot 98$ & 1.48 & - \\
\hline & $588 \cdot 0$ & $2 \cdot 29$ & $0 \cdot 39$ & $(1 \cdot 10)$ \\
\hline \multirow[t]{3}{*}{ Cholesterol $(\mathrm{mmol} / \mathrm{l})$} & $2 \cdot 56$ & $0 \cdot 03$ & $1 \cdot 23$ & $(2 \cdot 54)$ \\
\hline & $4 \cdot 83$ & $0 \cdot 06$ & $1 \cdot 32$ & $(2 \cdot 88)$ \\
\hline & $5 \cdot 82$ & 0.07 & $1 \cdot 22$ & - \\
\hline Triglyceride (mmol/l) & $0 \cdot 26$ & $0 \cdot 015$ & $5 \cdot 71$ & $(14 \cdot 2)$ \\
\hline & 0.78 & $0 \cdot 040$ & $5 \cdot 10$ & - \\
\hline & $2 \cdot 23$ & $0 \cdot 040$ & $1 \cdot 82$ & - \\
\hline
\end{tabular}

Reviewing the absorbance data obtained for each of the calibrators over the period of the evaluation showed significant variation in absorbance for $\mathrm{TCO}_{2}$, urea and calcium. 
Table 3. Between-batch precision at three analyte concentrations for controls analysed immediately following calibration. Results for controls analysed in the afternoon are given in parentheses.

\begin{tabular}{|c|c|c|c|c|}
\hline Analyte & Mean & $S D$ & $C V(\%)$ & $C V(\%)$ \\
\hline \multirow[t]{3}{*}{ Sodium $(\mathrm{mmol} / \mathrm{l})$} & $123 \cdot 4$ & $1 \cdot 14$ & 0.92 & $(1 \cdot 00)$ \\
\hline & $140 \cdot 6$ & $1 \cdot 65$ & $1 \cdot 17$ & $(0 \cdot 88)$ \\
\hline & $150 \cdot 0$ & $1 \cdot 45$ & $0 \cdot 97$ & $(1 \cdot 05)$ \\
\hline \multirow[t]{3}{*}{ Potassium (mmol/l) } & $1 \cdot 90$ & $0 \cdot 00$ & $0 \cdot 00$ & $(0 \cdot 00)$ \\
\hline & $4 \cdot 30$ & $0 \cdot 04$ & $0 \cdot 88$ & $(1 \cdot 40)$ \\
\hline & $6 \cdot 59$ & $0 \cdot 07$ & $1 \cdot 02$ & $(1 \cdot 29)$ \\
\hline \multirow[t]{3}{*}{ Chloride (mmol/l) } & $88 \cdot 0$ & 0.93 & 1.05 & $(1 \cdot 31)$ \\
\hline & $98 \cdot 9$ & $1 \cdot 13$ & $1 \cdot 14$ & $(1 \cdot 67)$ \\
\hline & $112 \cdot 1$ & $1 \cdot 19$ & 1.06 & $(1.90)$ \\
\hline \multirow[t]{3}{*}{$\mathrm{TCO}_{2}(\mathrm{mmol} / \mathrm{l})$} & $18 \cdot 9$ & $1 \cdot 29$ & $6 \cdot 90$ & $(10 \cdot 40)$ \\
\hline & $28 \cdot 0$ & $1 \cdot 65$ & $5 \cdot 91$ & $(8 \cdot 10)$ \\
\hline & $32 \cdot 8$ & $2 \cdot 07$ & $6 \cdot 32$ & $(9 \cdot 09)$ \\
\hline \multirow[t]{3}{*}{ Glucose $(\mathrm{mmol} / \mathrm{l})$} & $4 \cdot 12$ & $0 \cdot 12$ & $2 \cdot 56$ & $(2 \cdot 70)$ \\
\hline & $12 \cdot 41$ & $0 \cdot 38$ & 3.09 & $(2 \cdot 44)$ \\
\hline & $19 \cdot 91$ & $0 \cdot 43$ & $2 \cdot 16$ & $(2 \cdot 62)$ \\
\hline \multirow{3}{*}{ Urea $(\mathrm{mmol} / \mathrm{l})$} & 6.03 & $0 \cdot 19$ & $3 \cdot 10$ & $(4 \cdot 02)$ \\
\hline & $19 \cdot 90$ & 0.58 & $2 \cdot 91$ & $(2 \cdot 88)$ \\
\hline & $29 \cdot 59$ & $1 \cdot 14$ & $3 \cdot 84$ & $(5 \cdot 15)$ \\
\hline \multirow[t]{3}{*}{ Greatinine $(\mu \mathrm{mol} / \mathrm{l})$} & $106 \cdot 3$ & $8 \cdot 05$ & $7 \cdot 60$ & $(4 \cdot 70)$ \\
\hline & $452 \cdot 8$ & $8 \cdot 48$ & $1 \cdot 87$ & $(2 \cdot 38)$ \\
\hline & $718 \cdot 1$ & $11 \cdot 91$ & $1 \cdot 66$ & $(2 \cdot 59)$ \\
\hline \multirow[t]{3}{*}{ Total protein $(\mathrm{g} / \mathrm{l})$} & $39 \cdot 5$ & $0 \cdot 75$ & $1 \cdot 90$ & $(2 \cdot 88)$ \\
\hline & $67 \cdot 1$ & $1 \cdot 21$ & $1 \cdot 81$ & $(2 \cdot 52)$ \\
\hline & $82 \cdot 4$ & $1 \cdot 53$ & $1 \cdot 85$ & $(1 \cdot 77)$ \\
\hline \multirow[t]{3}{*}{ Albumin $(\mathrm{g} / \mathrm{l})$} & $24 \cdot 2$ & $0 \cdot 44$ & $1 \cdot 81$ & $(2 \cdot 63)$ \\
\hline & $38 \cdot 8$ & $1 \cdot 10$ & $2 \cdot 86$ & $(3 \cdot 19)$ \\
\hline & $49 \cdot 0$ & $0 \cdot 46$ & $0 \cdot 94$ & $(1 \cdot 80)$ \\
\hline \multirow[t]{3}{*}{ Calcium (mmol/l) } & $1 \cdot 03$ & $0 \cdot 058$ & $5 \cdot 59$ & $(3 \cdot 48)$ \\
\hline & $2 \cdot 48$ & $0 \cdot 042$ & $1 \cdot 70$ & $(3 \cdot 07)$ \\
\hline & $3 \cdot 25$ & $0 \cdot 050$ & 1.53 & $(2 \cdot 93)$ \\
\hline \multirow[t]{3}{*}{ Phosphate $(\mathrm{mmol} / \mathrm{l})$} & $1 \cdot 11$ & $0 \cdot 022$ & 1.95 & $(3 \cdot 13)$ \\
\hline & $1 \cdot 64$ & $0 \cdot 036$ & $2 \cdot 17$ & $(3 \cdot 21)$ \\
\hline & $2 \cdot 72$ & $0 \cdot 054$ & 1.99 & $(3 \cdot 37)$ \\
\hline \multirow[t]{3}{*}{ T Bilirubin $(\mu \mathrm{mol} / \mathrm{l})$} & $10 \cdot 8$ & $0 \cdot 83$ & $7 \cdot 70$ & $(6 \cdot 70)$ \\
\hline & $60 \cdot 0$ & $1 \cdot 50$ & $2 \cdot 50$ & $(4 \cdot 00)$ \\
\hline & $149 \cdot 9$ & $3 \cdot 72$ & $2 \cdot 48$ & $(2 \cdot 64)$ \\
\hline \multirow[t]{3}{*}{$\operatorname{ALP}(\mathrm{I} . \mathrm{U} . / \mathrm{l})$} & $80 \cdot 3$ & $2 \cdot 63$ & $3 \cdot 27$ & $(3 \cdot 37)$ \\
\hline & $176 \cdot 8$ & $5 \cdot 16$ & $2 \cdot 92$ & $(2 \cdot 24)$ \\
\hline & 653.9 & $15 \cdot 70$ & $2 \cdot 40$ & $(2 \cdot 21)$ \\
\hline \multirow[t]{3}{*}{$\operatorname{ALT}($ I.U./l) } & $22 \cdot 7$ & $1 \cdot 49$ & $6 \cdot 60$ & $(6 \cdot 40)$ \\
\hline & $80 \cdot 3$ & 1.92 & $2 \cdot 39$ & $(3 \cdot 27)$ \\
\hline & $192 \cdot 3$ & $3 \cdot 09$ & $1 \cdot 61$ & $(1 \cdot 58)$ \\
\hline \multirow[t]{3}{*}{ CK (I.U./l) } & $119 \cdot 6$ & $10 \cdot 15$ & $8 \cdot 50$ & $(8 \cdot 12)$ \\
\hline & $226 \cdot 1$ & $21 \cdot 30$ & $9 \cdot 41$ & $(11 \cdot 00)$ \\
\hline & $445 \cdot 8$ & $37 \cdot 04$ & $8 \cdot 31$ & $(5 \cdot 48)$ \\
\hline \multirow[t]{3}{*}{ LD (I.U./l) } & $236 \cdot 7$ & $7 \cdot 00$ & $2 \cdot 97$ & $(3 \cdot 20)$ \\
\hline & $392 \cdot 0$ & $16 \cdot 80$ & $4 \cdot 28$ & $(3 \cdot 57)$ \\
\hline & $631 \cdot 5$ & $20 \cdot 73$ & $3 \cdot 28$ & $(3 \cdot 11)$ \\
\hline \multirow[t]{3}{*}{ Urate $(\mu \mathrm{mol} / \mathrm{l})$} & $210 \cdot 0$ & $5 \cdot 22$ & $2 \cdot 48$ & $(4 \cdot 37)$ \\
\hline & $334 \cdot 3$ & $6 \cdot 87$ & $2 \cdot 05$ & $(3.96)$ \\
\hline & $583 \cdot 0$ & $11 \cdot 37$ & $1 \cdot 95$ & $(3 \cdot 76)$ \\
\hline \multirow[t]{3}{*}{ Cholesterol $(\mathrm{mmol} / \mathrm{l})$} & $2 \cdot 65$ & $0 \cdot 079$ & $3 \cdot 00$ & $(2 \cdot 85)$ \\
\hline & $4 \cdot 87$ & $0 \cdot 116$ & $2 \cdot 40$ & $(2 \cdot 40)$ \\
\hline & $6 \cdot 03$ & $0 \cdot 150$ & $2 \cdot 47$ & $(4 \cdot 29)$ \\
\hline Triglyceride $(\mathrm{mmol} / \mathrm{l})$ & $0 \cdot 62$ & $0 \cdot 050$ & $8 \cdot 13$ & $(4 \cdot 39)$ \\
\hline & $2 \cdot 11$ & $0 \cdot 097$ & $4 \cdot 62$ & $(2 \cdot 99)$ \\
\hline & $5 \cdot 56$ & $0 \cdot 190$ & $3 \cdot 38$ & $(2 \cdot 78)$ \\
\hline
\end{tabular}

Assessing calibration stability by calculation of the analyte concentration based on day 1 calibration figures showed a variation of greater than $4 \mathrm{SD}$ in the case of calcium, phosphate, total protein and urate.
Table 4. Linearity of assays performed on the Monarch.

\begin{tabular}{lc}
\hline Analyte & Determined range of linearity \\
\hline Sodium & $110-160 \mathrm{mmol} / 1$ \\
Potassium & $1-10 \mathrm{mmol} / 1$ \\
Chloride & $70-140 \mathrm{mmol} / 1$ \\
TCO $_{2}$ & $0-45 \mathrm{mmol} / 1$ \\
Glucose & $0-30 \mathrm{mmol} / 1$ \\
Urea & $0-35 \mathrm{mmol} / 1$ \\
Creatinine & $0-1500 \mu \mathrm{mol} / 1$ \\
Total protein & $0-130 \mathrm{~g} / 1$ \\
Albumin & $0-50 \mathrm{~g} / 1$ \\
Calcium & $0-2 \cdot 5 \mathrm{mmol} / 1$ \\
Phosphate & $0-4.5 \mathrm{mmol} / 1$ \\
Bilirubin & $0-500 \mu \mathrm{mol} / 1$ \\
ALP & $0-1500 \mathrm{IU} / 1$ \\
ALT & $0-450 \mathrm{IU} / 1$ \\
CK & $0-1000 \mathrm{IU} / 1$ \\
LD & $0-750 \mathrm{IU} / 1$ \\
Urate & $0-0 \cdot 9 \mathrm{mmol} / 1$ \\
Cholesterol & $0-10 \cdot 5 \mathrm{mmol} / 1$ \\
Triglyceride & $0-10 \cdot 5 \mathrm{mmol} / 1$ \\
\hline
\end{tabular}

\section{Linearity}

The linear range for each analyte is shown in table 4 . The results obtained agreed with the expected ranges for each analyte, except in the case of calcium. The results suggest that the assay is not linear above $3.0 \mathrm{mmol} / \mathrm{l}$.

\section{Accuracy}

The method comparison studies indicated a good agreement in the majority of cases (table 5 and figure 1). Small differences were attributable to the use of different calibration materials. Major discrepancies were found with amylase and alkaline phosphatase, due to the use of different methods, and with CK and LD. Experiments were carried out in an attempt to determine the cause of

Table 5. Linear regression statistics for Monarch (y-axis) against various comparison methods ( $x$-axis).

\begin{tabular}{|c|c|c|c|c|}
\hline Test & $N$ & Slope & $Y$-intercept & $\begin{array}{c}\text { Correlation } \\
\text { coefficient }\end{array}$ \\
\hline Sodium & 176 & $1 \cdot 126$ & $-16 \cdot 14$ & $0 \cdot 987$ \\
\hline Potassium & 148 & 0.979 & $0 \cdot 357$ & 0.996 \\
\hline $\mathrm{TCO}_{2}$ & 121 & $0 \cdot 925$ & $-4 \cdot 37$ & $0 \cdot 837$ \\
\hline Glucose & 161 & 0.975 & $-0 \cdot 049$ & $0 \cdot 997$ \\
\hline Urea & 188 & $1 \cdot 012$ & $0 \cdot 60$ & $0 \cdot 996$ \\
\hline Creatinine & 170 & 0.926 & $25 \cdot 57$ & $0 \cdot 997$ \\
\hline Total protein & 137 & $0 \cdot 928$ & $4 \cdot 33$ & $0 \cdot 974$ \\
\hline Albumin & 105 & 0.933 & $3 \cdot 057$ & $0 \cdot 984$ \\
\hline Calcium & 139 & 0.981 & $0 \cdot 035$ & $0 \cdot 962$ \\
\hline Phosphate & 123 & $1 \cdot 025$ & $-0 \cdot 068$ & $0 \cdot 980$ \\
\hline Total bilirubin & 161 & 0.975 & -0.479 & 0.997 \\
\hline ALP & 156 & 1.571 & $-0 \cdot 874$ & $0 \cdot 989$ \\
\hline $\mathrm{ALT}$ & 145 & 0.925 & -0.057 & $0 \cdot 994$ \\
\hline CK & 161 & $0 \cdot 827$ & $0 \cdot 568$ & $0 \cdot 999$ \\
\hline $\mathrm{LD}$ & 160 & $0 \cdot 725$ & $17 \cdot 27$ & $0 \cdot 996$ \\
\hline Urate & 118 & 0.935 & $-0 \cdot 002$ & $0 \cdot 985$ \\
\hline Cholesterol & 141 & $1 \cdot 095$ & $0 \cdot 069$ & $0 \cdot 990$ \\
\hline Triglyceride & 114 & $0 \cdot 991$ & $-0 \cdot 04$ & $0 \cdot 995$ \\
\hline
\end{tabular}



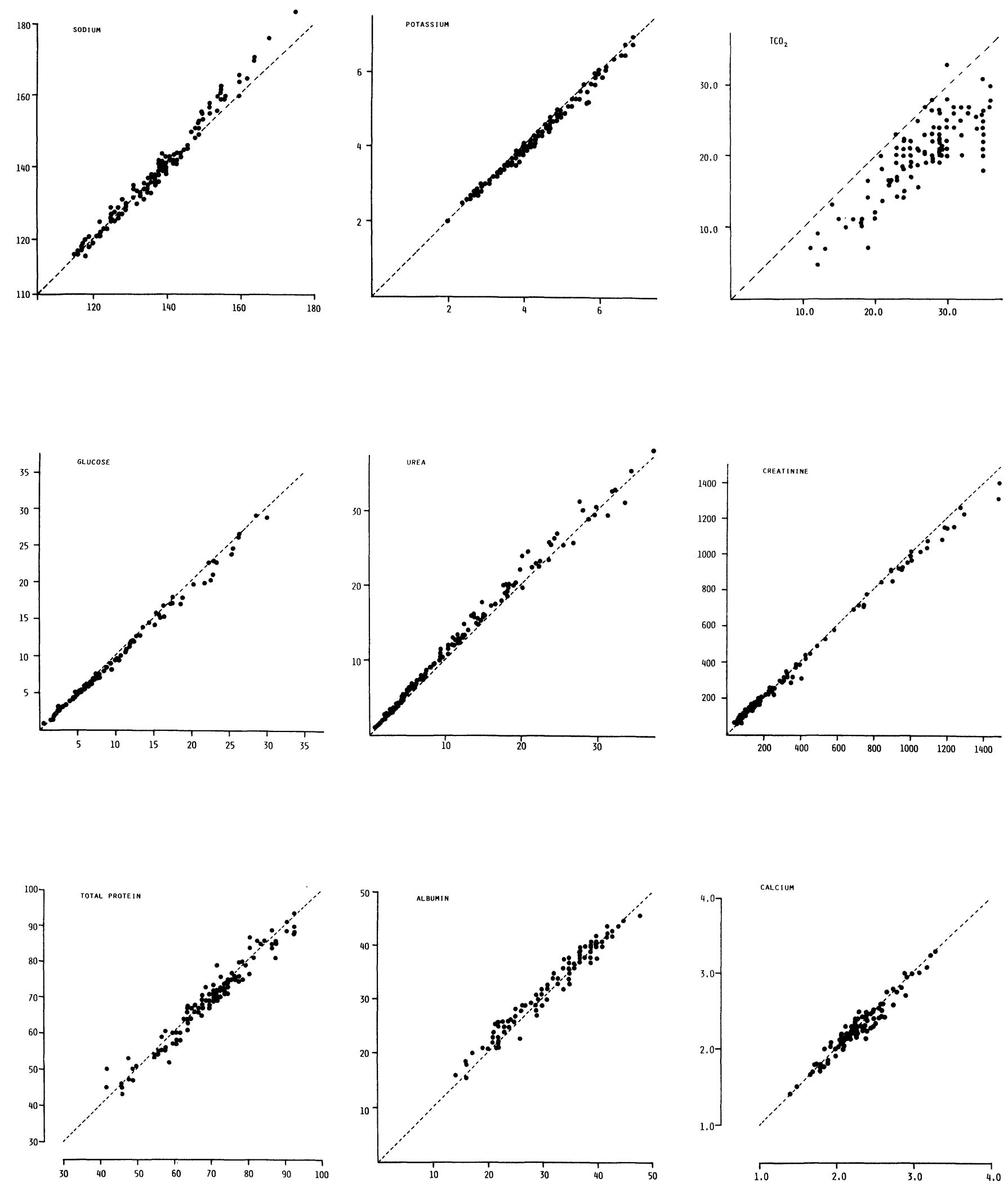

Figure 1. Comparison of results for analytes measured on the Monarch (y-axis) and comparison method (x-axis). Details of comparison methods are given in table 1. 

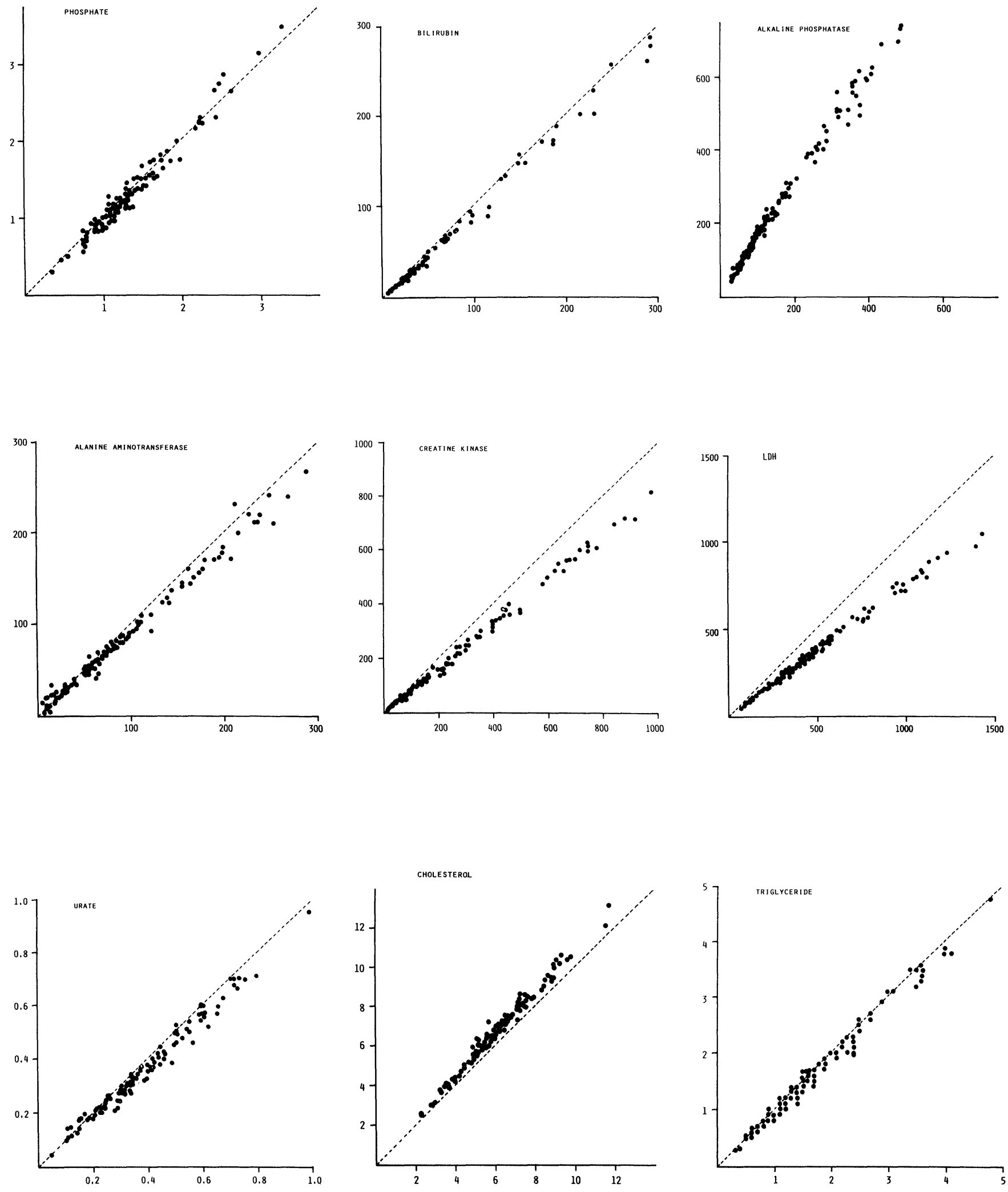

Figure 1 continued. 
Table 6. Experiment to determine recovery of aqueous and serum based sample pipetting on the Monarch.

\begin{tabular}{lccc}
\hline Sample & $\begin{array}{c}\text { *Measured } \\
\text { absorbance }\end{array}$ & $\begin{array}{c}\text { Expected } \\
\text { absorbance }\end{array}$ & $\begin{array}{c}\text { Recovery } \\
(\%)\end{array}$ \\
\hline $\begin{array}{l}\text { NADH: } \\
(\mu \mathrm{l})\end{array}$ & & & \\
3 & & & \\
6 & $0 \cdot 1863$ & $0 \cdot 1922$ & 97 \\
9 & 0.3602 & 0.3817 & 94 \\
12 & 0.5367 & 0.5739 & 94 \\
& 0.7184 & 0.7567 & 95 \\
Glucose in 60\% Albumin: & & \\
Concentration & & & \\
(mmol/l) & & & \\
5 & 0.344 & 0.403 & 85 \\
10 & 0.664 & 0.806 & 84 \\
15 & 1.069 & $1 \cdot 209$ & 88 \\
\hline
\end{tabular}

* NADH: mean value of five determinations. Glucose: mean value of three determinations.

Table 7. Sample to sample carry-over on the Monarch.

\begin{tabular}{lcccc}
\hline & & & & Mean \\
Analyte & Mean high & Mean low \\
level $($ H3) & level $($ L3) & Mean & L1-L3 & $\begin{array}{c}\text { carry-over } \\
(\%)\end{array}$ \\
\hline Sodium $(\mathrm{mmol} / \mathrm{l})$ & $258 \cdot 1$ & $99 \cdot 5$ & $0 \cdot 1$ & $0 \cdot 06$ \\
Potassium $(\mathrm{mmol} / \mathrm{l})$ & $12 \cdot 8$ & $1 \cdot 9$ & 0 & 0 \\
Chloride $(\mathrm{mmol} / \mathrm{l})$ & $180 \cdot 8$ & $91 \cdot 8$ & $1 \cdot 7$ & $1 \cdot 9$ \\
TCO $(\mathrm{mmol} / \mathrm{l})$ & $57 \cdot 6$ & $3 \cdot 8$ & $0 \cdot 4$ & $0 \cdot 70$ \\
Glucose $(\mathrm{mmol} / \mathrm{l})$ & $35 \cdot 5$ & $2 \cdot 1$ & $0 \cdot 1$ & $0 \cdot 30$ \\
Urea $(\mathrm{mmol} / \mathrm{l})$ & $44 \cdot 8$ & $2 \cdot 6$ & $0 \cdot 2$ & $0 \cdot 47$ \\
Creatinine $(\mu \mathrm{mol} / \mathrm{l})$ & 1246 & 58 & 3 & $0 \cdot 25$ \\
Total protein $(\mathrm{g} / \mathrm{l})$ & $136 \cdot 8$ & $30 \cdot 3$ & $0 \cdot 6$ & $0 \cdot 56$ \\
Albumin $(\mathrm{g} / \mathrm{l})$ & $42 \cdot 2$ & $15 \cdot 5$ & $0 \cdot 3$ & $1 \cdot 12$ \\
Calcium $(\mathrm{mmol} / \mathrm{l})$ & $4 \cdot 29$ & $1 \cdot 20$ & $0 \cdot 01$ & $0 \cdot 32$ \\
Phosphate $(\mathrm{mmol} / \mathrm{l})$ & $5 \cdot 30$ & $0 \cdot 34$ & $0 \cdot 01$ & $0 \cdot 20$ \\
Total bilirubin & & & & \\
$\quad(\mu \mathrm{mol} / \mathrm{l})$ & 823 & $4 \cdot 8$ & $0 \cdot 40$ & $0 \cdot 05$ \\
ALP $(\mathrm{IU} / \mathrm{l})$ & 1200 & 52 & $1 \cdot 00$ & $0 \cdot 09$ \\
ALT $(\mathrm{IU} / \mathrm{l})$ & 2312 & 18 & $5 \cdot 0$ & $0 \cdot 22$ \\
CK $(\mathrm{IU} / \mathrm{l})$ & 4000 & 43 & $2 \cdot 0$ & $0 \cdot 05$ \\
LD $(\mathrm{IU} / \mathrm{l})$ & 1360 & 103 & $2 \cdot 0$ & $0 \cdot 16$ \\
Urate $(\mu \mathrm{mol} / \mathrm{l})$ & $1 \cdot 05$ & $0 \cdot 152$ & $0 \cdot 005$ & $0 \cdot 56$ \\
Cholesterol $(\mathrm{mmol} / \mathrm{l})$ & $11 \cdot 08$ & $1 \cdot 27$ & $0 \cdot 06$ & $0 \cdot 61$ \\
Triglyceride $(\mathrm{mmol} / \mathrm{l})$ & $10 \cdot 0$ & $0 \cdot 74$ & $0 \cdot 07$ & $0 \cdot 76$ \\
\hline
\end{tabular}

the discrepancy found with GK and LD assays. Solutions of NADH were prepared and loaded by the instrument using 3, 6, 9 and $12 \mu \mathrm{l}$ sample volumes. The experiment was also carried out using 5,10 and $15 \mathrm{mmol} / 1$ glucose in $60 \%$ albumin using an end-point glucose dehydrogenase method adapted to the Monarch. The expected absorbances (determined from the extinction coefficient of $\mathrm{NADH})$ at $340 \mathrm{~nm}$ were confirmed with similar experiments using externally, manually loaded rotors (table 6).

\section{System carry-over}

Sample carry-over - the mean carry-over obtained in all cases was negligible (table 7).

Reagent carry-over - the coefficient of variation obtained was less than 4SD (mean value obtained in the withinbatch precision study) in all cases except two. The experiment was therefore repeated using three sequences of the initial procedures, for $\mathrm{CK}$ into creatinine and triglyceride into LD. No significant carry-over was detected.

\section{Conclusion}

The Monarch chemistry analyser demonstrated good performance over a wide range of analytes. The coefficients of variation obtained for the within-batch and between-day precision data were good, although the performance of the $\mathrm{TCO}_{2}$ and calcium methods were disappointing.

The linearity of the methods were sufficiently broad to allow measurements over a wide range without the necessity to dilute samples. There was no significant carry-over.

For the majority of the methods calibration would only be required once a day, and, in many cases, weekly calibration would be acceptable.

Comparison between the Monarch methods and those used in the routine laboratory were good in the majority of cases. The discrepancy seen in the enzyme results could not be resolved and it is currently necessary to employ a factor to correct for the difference.

The Monarch is capable of handling a reasonably large and varied workload. The work organization of the instrument is most efficient when batches of tests are analysed together. In this way a discretionary approach to testing can be achieved without affecting the performance of the instrument. Stat samples can be given priority at any time during a routine run.

We found the instrument to be flexible and easy to use, requiring a minimum of training. Only simple maintenance procedures were required on a daily, weekly and monthly basis.

\section{References}

1. Anderson, N. G., American Journal of Clinical Pathology, $\mathbf{5 3}$ (1970), 778.

2. Price, C. P. and Spencer, K. (Eds) In Centrifugal Analysers in Clinical Chemistry, edited by Price, C. P. and Spencer, K. (Praeger, Eastbourne, 1980), p. 5.

3. Tiffany, T. O. In Centrifugal Analysers in Clinical Chemistry, edited by Price, C. P. and Spencer, K. (Praeger, Eastbourne, 1980), p. 3.

4. Broughton, P. M. G., Gowenlock, A. H., MaCormack, J. J. and NeILl, D. W., Annals of Clinical Biochemistry, 11 (1974), 207. 


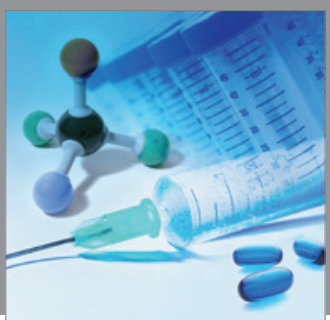

International Journal of

Medicinal Chemistry

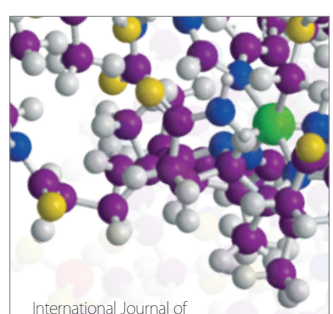

Carbohydrate Chemistry

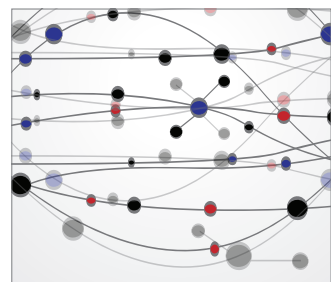

The Scientific World Journal
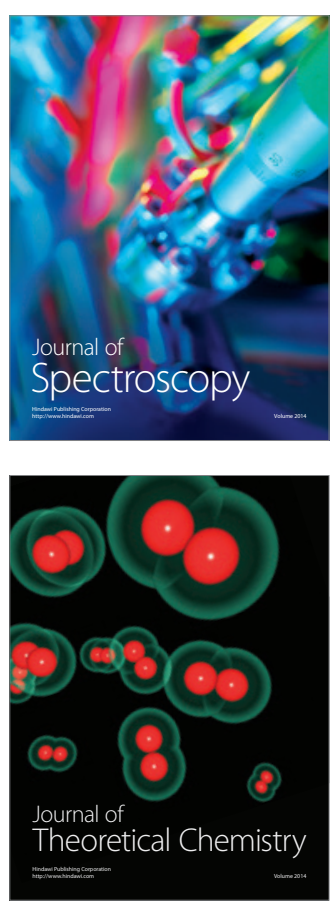
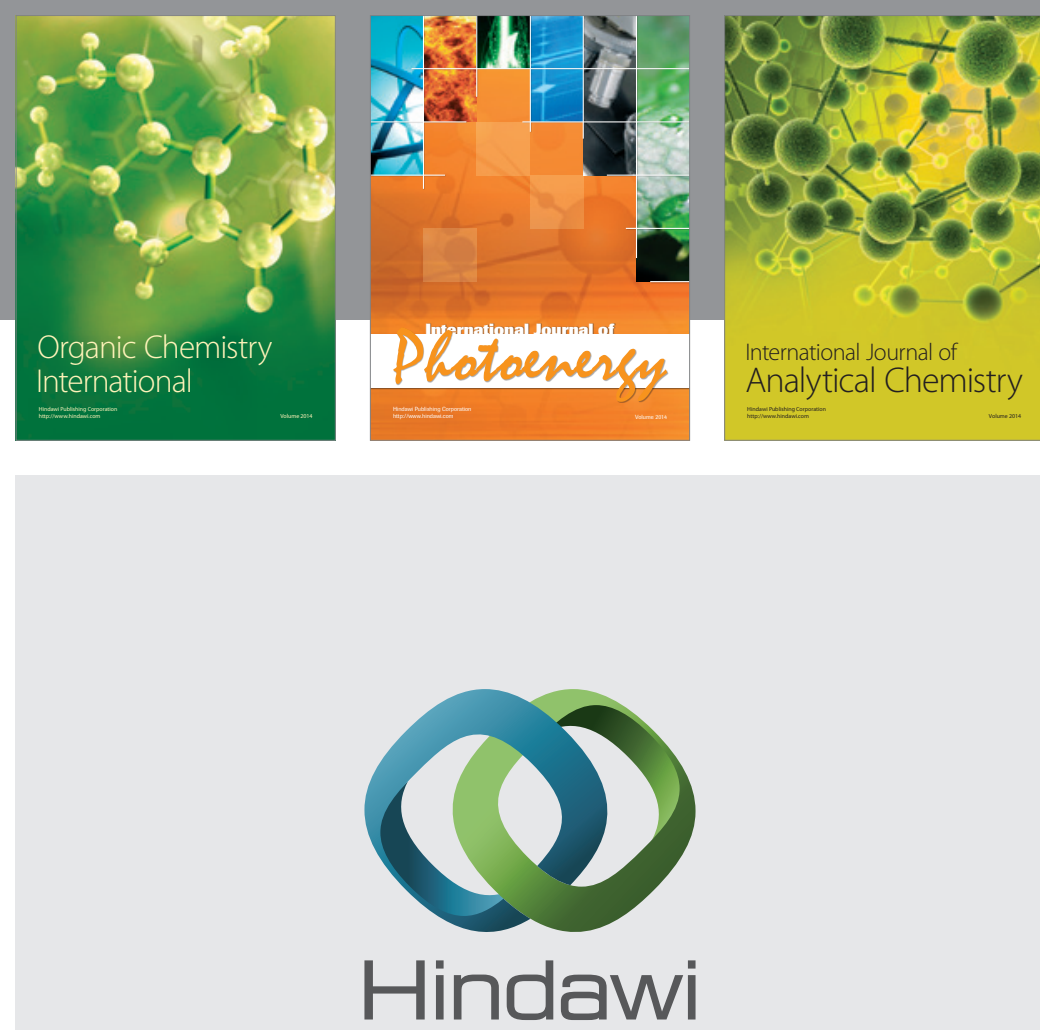

Submit your manuscripts at

http://www.hindawi.com
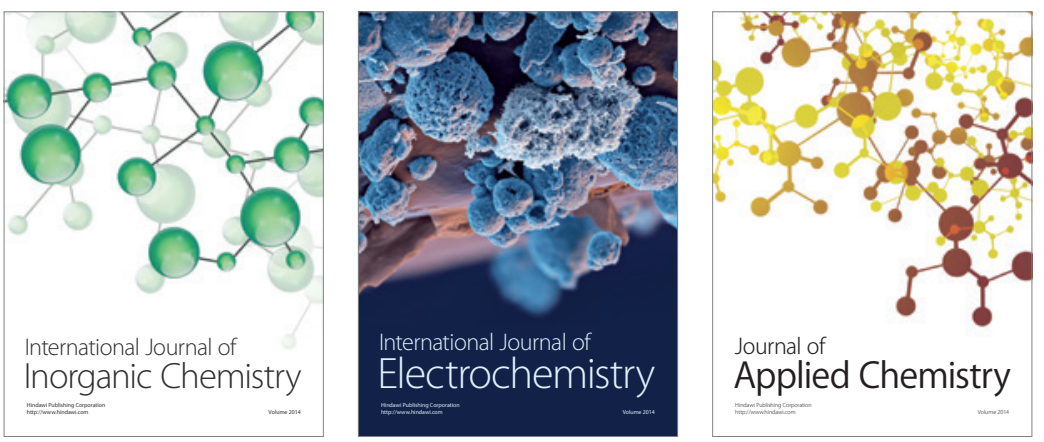

Journal of

Applied Chemistry
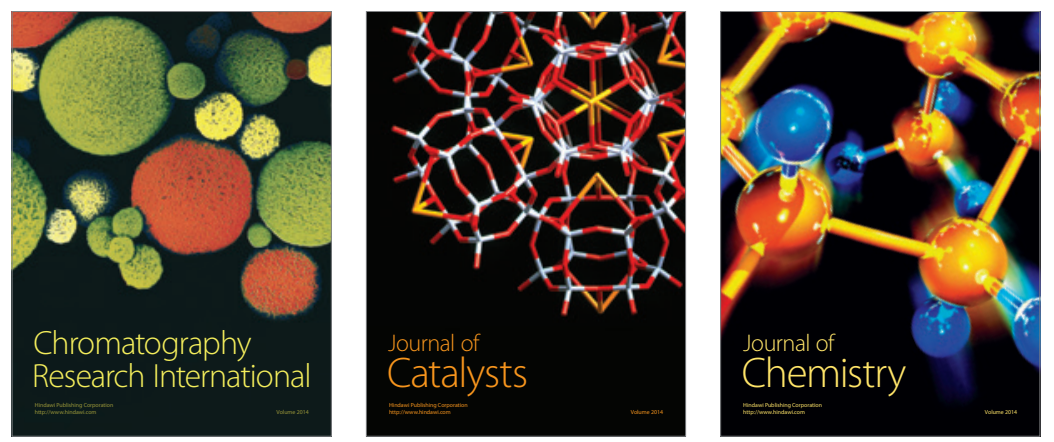
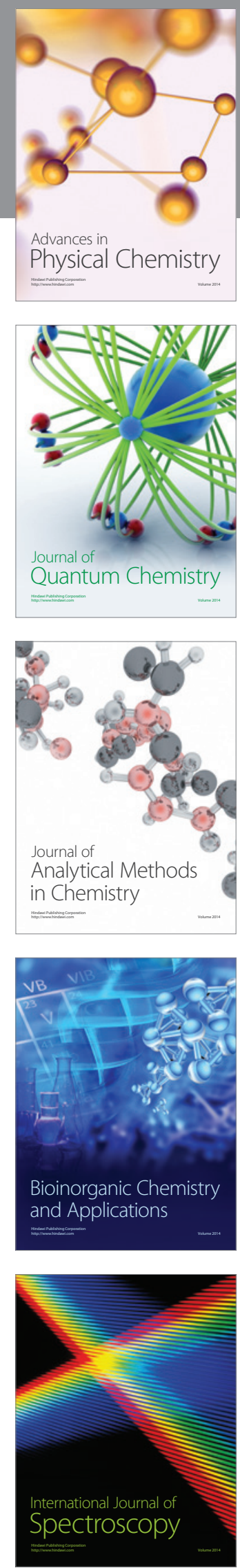